\title{
¿Protege o predispone la actividad física a las adicciones conductuales y otros problemas de autorregulación?
}

\section{Exploring the direct or inverse association of physical activity with behavioral addictions and other self-regulation problems}

\author{
Cinderella K. Cardol, MSc*, Cristopher I. Escamilla, MSc*, Winifred A. Gebhardt, PhD*, \\ José C. Perales, PhD**. \\ * Health, Medical and Neuropsychology Unit; Leiden University. \\ ** Department of Experimental Psychology; Mind, Brain and Behavior Research Center (CIMCYC); University of Granada.
}

\section{Resumen}

Este estudio tuvo como objetivo aclarar las relaciones entre la actividad física de ocio (AFO) y los problemas de autorregulación (PARs) no relacionados con drogas, incluyendo las adicciones conductuales, y el papel de los rasgos de personalidad impulsiva. Estudiantes universitarios españoles $\left(N=329, M_{\text {age }}=21,20\right)$ completaron cuestionarios para cada uno de estos constructos.

La AFO de gimnasio y musculación se asoció negativamente con los PARs relacionados con videojuegos, $r=-0,13, p=0,019,95 \%$ CI (bootstrapped) [-0,23, -0,02], y positivamente con los PARs relacionados con sexo, $r=0,16, p=0,005,95 \%$ CI (bootstrapped) [0,04, -0,30]. La AFO de resistencia se asoció positivamente con los PARs relacionados con la alimentación, $r=0,17, p=0,003,95 \%$ CI (bootstrapped) [0,02, 0,31]. La probabilidad de presentar puntuaciones potencialmente clínicas en PARs de alimentación fue 2,64 veces mayor para los encuestados en un grupo de AFO de resistencia excesiva en comparación con los otros encuestados, prueba exacta de Fisher, $p=0,017$, OR $=3,10,95 \%$ CI $[1,26,7,63]$, y la probabilidad de vomitar para controlar el peso fue 2,12 veces mayor en ese mismo grupo, prueba exacta de Fisher, $p$ $=0,040, \mathrm{OR}=2,43,95 \%$ CI $[1,06,5,57]$. Las asociaciones fueron en gran medida independientes de los rasgos de personalidad impulsiva. Identificamos un riesgo elevado de patología alimentaria potencialmente clínica en un subgrupo de participantes con niveles anormalmente altos de actividad física de resistencia. Esta superposición es consistente con la hipótesis de dependencia secundaria en la adicción al ejercicio

Palabras clave: Actividad física de ocio; Ejercicio físico; Adicción al ejercicio; Autorregulación; Adicción conductual; Impulsividad; Rasgos de personalidad impulsiva.

\begin{abstract}
This cross-sectional survey study had the aim of clarifying the relationships between leisure time physical activity (LTPA) and non-drugrelated self-regulation problems (non-drug-related SRPs), including behavioral addictions, and the role of impulsive personality traits therein. Spanish university students $\left(N=329 ; M_{\text {age }}=21.20\right)$ completed questionnaires for each of these constructs.

Fitness and Bodybuilding LTPA was negatively associated with video gaming-related SRPs, $r=-.13, p=.019,95 \%$ CI (bootstrapped) $[-.23$, $-.02]$, and positively associated with sex-related SRPs, $r=.16, p=.005$, 95\% CI (bootstrapped) $[.04, .30]$. Endurance LTPA was associated with higher scores in eating-related SRPs, $r=.17, p=.003,95 \% \mathrm{CI}$ (bootstrapped) [.02, .31]. The proportion of participants presenting scores above the clinically significant threshold in eating-related SRPs was 2.64 times higher for respondents in an Excessive Endurance LTPA cluster compared to the other respondents, Fisher's exact test, $p=.017, \mathrm{OR}=3.10,95 \% \mathrm{CI}[1.26,7.63]$, and the proportion of participants reporting vomiting to control weight was 2.12 times higher, Fisher's exact test, $p=.040, \mathrm{OR}=2.43,95 \%$ CI $[1.06,5.57]$. The associations were largely independent of impulsive personality traits.

We identified an elevated risk of eating pathology in a subgroup of participants with anomalously high participation in endurance physical activity. This overlap is consistent with the secondary dependence hypothesis of exercise addiction.

Keywords: Leisure time physical activity; Physical exercise; Exercise addiction; Self-regulation; Behavioral addiction; Impulsivity; Impulsive personality traits.
\end{abstract}

Recibido: Diciembre 2016; Aceptado: Octubre 2017

Enviar correspondencia a:

Cinderella K. Cardol

E-mail: c.k.cardol@fsw.leidenuniv.nl. 


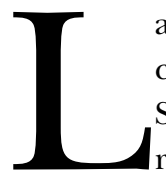

a actividad física (AF) se reconoce ampliamente como beneficiosa para la salud mental y física. Sin embargo, existe un debate de larga duración respecto a los riesgos potenciales de la participación en actividades deportivas. Más concretamente, la AF anormalmente intensa o frecuente muestra paralelismos con los problemas de autorregulación (PARs; Bratland-Sanda et al., 2011). La autorregulación requiere el control de los hábitos, impulsos y deseos, por lo que los comportamientos mal autorregulados pueden interferir con el bienestar (Vohs y Baumeister, 2011). Algunos de estos PARs, incluyendo la AF excesiva, han sido conceptualizados como adicciones conductuales (e.g., Grant, Potenza, Weinstein, y Gorelick, 2010).

Por lo tanto, la AF puede ser utilizada como una estrategia de intervención para mejorar la salud mental, pero, a la vez, determinados patrones de AF podrían solaparse con o ser un desencadenante de PARs. Con esta doble perspectiva en mente, exploramos las relaciones potenciales entre la cantidad de tiempo de ocio dedicado a la actividad física (AFO) y las quejas subjetivas asociadas a problemas de autorregulación (PARs) no relacionados con drogas, así como la posible implicación de rasgos de personalidad impulsiva en estas relaciones.

La mayoría de los PARs, así como determinados patrones de AF, se han asociado a los rasgos de personalidad impulsiva (Evenden, 1999; Joseph, Alonso-Alonso, Bond, Pascual-Leone, \& Blackburn, 2011; Knezevic-Budisin, Pedden, White, Miller, \& Hoaken, 2015; Lejoyeux, Tassain, Solomon, \& Adès, 1997; Perry \& Carroll, 2008; Raymond, Coleman, \& Miner, 2003; Verdejo-García, Lawrence, \& Clark, 2008). Según el modelo de Urgency, Premeditation, Perseveran$c e$, Sensation Seeking (UPPS-P [Urgencia, Búsqueda de Sensaciones, Falta de Premeditación, Falta de Perseverancia]; Whiteside y Lynam, 2001), la impulsividad se compone de: (a) urgencia negativa, p. ej., la tendencia a reaccionar de forma precipitada bajo la influencia de emociones negativas; (b) urgencia positiva, p. ej., la tendencia a actuar precipitadamente bajo estados emocionales positivos intensos; (c) búsqueda de sensaciones, p. ej., la tendencia a perseguir actividades nuevas y emocionantes; (d) falta de premeditación, p. ej., la tendencia a no pensar en las consecuencias de una acción antes de participar en ella; y (e) falta de perseverancia, p. ej., la incapacidad de permanecer centrado en tareas que exigen atención (Cyders et al., 2007).

Los rasgos de personalidad impulsiva pueden desempeñar diferentes funciones en las relaciones entre la AFO y los PARs no relacionados con drogas. Por un lado, el modelo de la fuerza del autocontrol $l^{1}$ predice que la disponibilidad de los

1 El autocontrol es un componente de la autorregulación (la capacidad de regular el comportamiento para conseguir los objetivos a largo plazo), es decir, el mecanismo cognitivo de arriba hacia abajo mediante el cual se inhiben los impulsos, hábitos y deseos. recursos de autocontrol subyace a los bajos niveles de rasgos de personalidad impulsiva, facilita la autorregulación y evita los PARs (Baumeister y Tierney, 2011; Baumeister, Vohs, y Tice, 2007). De acuerdo con esta predicción, los individuos con recursos reducidos de autocontrol son más propensos a cometer actos impulsivos y a mostrar PARs (Billieux, Gay, Rochat, y Van der Linden, 2010; Verdejo-García et al., 2008; Wills y Dishion, 2004; Wills, Iasi, Don Mendoza, y Ainette, 2007). Conjuntamente, un alto nivel de autocontrol se asocia con un bajo nivel de conducta sedentaria y con altos niveles de participación y rendimiento en AF, a través de una mayor tolerancia a la fatiga y una orientación hacia los beneficios a largo plazo (Dorris, Power, y Kenefick, 2012; Joseph et al., 2011; Wills et al., 2004; Wills et al., 2007). En otras palabras, los niveles altos de autocontrol (reflejados por bajos niveles de rasgos de personalidad impulsiva) podrían predecir tanto la implicación en la AFO como la disminución del riesgo de PARs no relacionados con drogas.

Por su parte, la hipótesis de dependencia a la AF concibe algunos casos de participación en deportes anormalmente alta como casos de un tipo específico de PAR, la llamada dependencia primaria a la AF (i.e., adicción al ejercicio). Alternativamente, la AF puede ser un estrategia para afrontar las consecuencias de otros problemas clínicos o subclínicos: la llamada dependencia secundaria a la AF (de Coverley Veale, 1987; Landolfi, 2013). En este sentido, los individuos dependientes de la AF practicarían AF rutinariamente en exceso $^{2}$, a menudo ignorando las lesiones y descuidando el trabajo, la familia y los amigos (de Coverley Veale, 1987). Se informa sobre esta condición con frecuencia entre los corredores y otros deportistas de resistencia (Allegre, Souville, Therme, y Griffiths, 2006; Breuer y Kleinert, 2009). Suponiendo que se considerase como un PAR primario, sería de esperar que la AF excesiva presentara cierto nivel de co-ocurrencia con otros comportamientos problemáticos en esta categoría. Se observa este tipo de solapamiento, por ejemplo, entre el abuso de sustancias y el juego de azar problemático (Navas, Torres, Vilar, et al., 2014; Petry, Stinson, y Grant, 2005) o entre el consumo perjudicial de alcohol y el uso excesivo de internet (Navas, Torres, Cándido, y Perales, 2014; Yen, Ko, Yen, Chen, y Chen, 2009). Extendiendo el paralelismo, se esperaría que por lo menos algunos deportistas excesivos (en los cuales el ejercicio excesivo se asemeja a un patrón adictivo) presentaran características de impulsividad similar a las observadas en

2 No existe consenso sobre la existencia de dependencia a la AF. Por ello, no existe una categoría diagnóstica en el Diagnostic and Statistical Manual of Mental Disorders (5th ed.; DSM-5; American Psychiatric Association, 2013). En realidad, el DSM-5 sólo reconoce el trastorno del juego patológico como una adicción comportamental (sin sustancia). Con el fin de evitar la controversia, utilizamos el término "AFO excesiva” para los casos de presentación de niveles muy elevados de participación en AFO en comparación con valores medios de AFO, de una forma puramente descriptiva y sin ninguna connotación clínica. 
otros comportamientos adictivos, con la búsqueda de sensaciones como señal de exposición temprana, y la urgencia negativa como un predictor de trastorno conductual (Billieux et al., 2007; Dick et al., 2010; Navas, Torres, Cándido, y Perales, 2014; Navas, Torres, Vilar, et al., 2014).

De particular interés es la relación específica entre la conducta alimentaria desregulada y la AFO de duración excesiva (Grandi, Clementi, Guidi, Benassi, y Tossani, 2011; Lichtenstein, Christiansen, Elklit, Bilenberg, y Støving, 2014). En los supuestos casos de dependencia primaria a la $\mathrm{AF}$, se esperaría que seguir una dieta y perder peso fueran estrategias para incrementar el rendimiento, mientras que la dependencia secundaria a la $\mathrm{AF}$ puede ocurrir entre personas con trastornos de conducta alimentaria, donde la AF es un medio para perder peso (Adams y Kirkby, 2001; de Coverley Veale, 1987).

Nuestro objetivo general de investigación era explorar las relaciones potenciales entre los patrones de práctica de diferentes formas de AFO y PARs no relacionados con drogas (incluyendo las adicciones conductuales) y el rol que los rasgos de personalidad impulsiva juegan en estas relaciones. Existen pocos estudios sobre las relaciones entre la AFO y PARs, más allá del abuso de sustancias (e.g., Lisha y Sussman, 2010). Además, la importancia de las modalidades de $\mathrm{AF}$ aún no ha sido exhaustivamente considerada (Allegre et al., 2006; Breuer et al., 2009; Ziemainz et al., 2013). Sin embargo, los deportistas que practican distintos tipos de AFO se diferencian sobre todo por su motivación y objetivos personales relativos al ejercicio (Ford, 2007; Kondric et al., 2013; NIH, 2011), y se ha encontrado que estos motivos subyacentes pueden influenciar las asociaciones con PARs relacionados con drogas (Kondric et al., 2011). Teniendo en cuenta la ausencia de evidencia directa, nuestras expectativas sobre la dirección de las relaciones permanecen abiertas. Una asociación en la que una puntuación baja en rasgos de personalidad impulsiva contribuyese simultáneamente a una probabilidad mayor de participación en AFO y a una probabilidad menor de presentar PARs no relacionados con drogas estaría en consonancia con el modelo de la fuerza del autocontrol (Baumeister et al., 2011; Baumeister et al., 2007). Por el contrario, una relación positiva y directa de la AFO de resistencia con PARs no relacionados con drogas así como con rasgos de personalidad impulsiva apoyaría la hipótesis de dependencia de AF. Más concretamente, una asociación entre PARs potencialmente clínicos relacionados con la alimentación y no relacionados con drogas y AFO excesiva apoyaría la hipótesis de dependencia secundaria a la AF (de Coverley Veale, 1987).

\section{Métodos}

\section{Participantes}

Los participantes fueron estudiantes de la Universidad de Granada, España. Ser un estudiante universitario era el único criterio de inclusión. Para conseguir el tamaño de la muestra necesario para el análisis estadístico, la muestra definitiva se compuso de 329 estudiantes con edades entre 18 y 41 años $\left(M_{\text {edad }}=21,22, D T=3,50\right)$. La muestra incluyó a 183 mujeres y 146 varones. Los participantes estudiaban grados en Ciencias del Deporte $(n=125)$, Psicología $(n=$ $116)$, Sociología $(n=42)$, Logopedia $(n=18)$, un Máster $(n=4)$ u otros $(n=22)$. La mayoría de los participantes $(n$ = 227) estaban en el primer año de su grado.

\section{Diseño y Procedimiento}

Para este estudio transversal, reclutamos a los participantes mediante muestreo de conveniencia en diferentes facultades de la Universidad de Granada, sin ofrecer ninguna compensación. Todos los participantes recibieron información acerca de los objetivos del estudio y dieron su consentimiento informado antes de la participación. Los participantes completaron una serie de cuestionarios de autoinforme de lápiz y papel en clase. Las instrucciones fueron proporcionadas por miembros del equipo de investigación. El procedimiento fue aprobado por el Comité de Ética del Universidad de Granada (Vicerrectorado de Política Científica e Investigación) con el número de referencia 2014-901.

\section{Medidas}

Medimos la AFO a través del Cuestionario sobre la Participación en Actividades Deportivas por Tipo (CPAD-T [Questionnaire of Participation in Sports Activities per Type]). Adaptamos el "Global Physical Activity Questionnaire” (GPAQ [Cuestionario de Actividad Física Global]) de la Organización Mundial de la Salud para medir los patrones de participación en la AFO (Armstrong y Bull, 2006; Bull, Maslin, y Armstrong, 2009), discriminando entre tipos de deportes. La escala contiene las 40 AFOs más practicados en España según los resultados de una encuesta realizada por el Consejo Superior de Deportes del Gobierno Español (García y Llopis, 2011) y la opción de "otras actividades físicas, a saber...”. Basado en el sistema de medición del GPAQ, los encuestados debían indicar qué AFOs practican actualmente, cuántos días a la semana practicaron cada actividad durante una semana típica y cuántas horas y minutos en un día típico: un día en que practican una determinada AFO. Los estudiantes de la Facultad de Ciencias del Deporte rellenaron el cuestionario dos veces, para separar las AFs académicas de las actividades que practican en su tiempo de ocio. Aquí, sólo utilizamos las últimas para los análisis, ya que nuestro enfoque era la práctica de tiempo libre, y la $\mathrm{AF}$ obligatoria podría distorsionar los resultados. El GPAQ se utiliza extensamente en el campo científico y posee buenas propiedades psicométricas (Armstrong et al., 2006).

Para reducir el número de AFOs evaluadas con el cuestionario CPAD-T a un conjunto manejable de dimensiones, se extrajo la combinación de dimensiones que mejor explicaba las correlaciones entre las diferentes AFOs con un 
Tabla 1. Pesos Factoriales del Análisis de Componentes Principales con Rotación Varimax para Actividades Físicas de Ocio (AFO) del Cuestionario de Participación en las Actividades Deportivas por Tipo ( $\mathrm{N}=329)$

\begin{tabular}{|c|c|c|c|c|c|c|c|}
\hline \multirow[b]{2}{*}{ AFO } & \multicolumn{7}{|c|}{ Peso factorial rotado } \\
\hline & 1 & 2 & 3 & 4 & 5 & 6 & 7 \\
\hline Pádel & 0,73 & 0,04 & $-0,02$ & $-0,00$ & 0,01 & 0,17 & 0,14 \\
\hline Tenis & 0,66 & $-0,16$ & $-0,00$ & $-0,07$ & 0,08 & 0,13 & 0,20 \\
\hline Fútbol & 0,60 & 0,19 & 0,02 & 0,16 & $-0,16$ & $-0,27$ & $-0,20$ \\
\hline Deportes de invierno & 0,00 & 0,76 & 0,08 & $-0,03$ & 0,08 & 0,10 & 0,25 \\
\hline Patinaje & 0,01 & 0,73 & $-0,08$ & 0,01 & $-0,07$ & $-0,05$ & $-0,21$ \\
\hline Gimnasia en casa & 0,02 & $-0,20$ & 0,73 & $-0,17$ & 0,08 & $-0,06$ & $-0,21$ \\
\hline Correr & 0,05 & 0,07 & 0,61 & 0,36 & $-0,23$ & 0,06 & 0,08 \\
\hline Senderismo & $-0,17$ & 0,32 & 0,58 & $-0,03$ & 0,06 & 0,04 & 0,46 \\
\hline Culturismo & 0,00 & $-0,13$ & 0,00 & 0,79 & $-0,13$ & 0,06 & $-0,02$ \\
\hline Gimnasia en el gimnasio & $-0,12$ & 0,18 & $-0,01$ & 0,63 & 0,42 & $-0,12$ & 0,03 \\
\hline Ejercicios aeróbicos & $-0,16$ & $-0,11$ & $-0,09$ & 0,11 & 0,72 & $-0,02$ & 0,10 \\
\hline Baloncesto & $-0,18$ & $-0,09$ & $-0,06$ & 0,13 & $-0,59$ & $-0,01$ & 0,16 \\
\hline Atletismo & 0,05 & $-0,06$ & $-0,12$ & 0,05 & $-0,10$ & 0,82 & 0,07 \\
\hline Ciclismo & 0,16 & 0,24 & 0,29 & $-0,06$ & 0,12 & 0,58 & $-0,22$ \\
\hline Natación & 0,28 & $-0,03$ & $-0,06$ & 0,01 & $-0,07$ & $-0,05$ & 0,75 \\
\hline Autovalor inicial & 1,75 & 1,60 & 1,30 & 1,24 & 1,15 & 1,05 & 1,03 \\
\hline$\%$ de la varianza & 11,68 & 10,65 & 8,64 & 8,24 & 7,66 & 6,98 & 6,87 \\
\hline
\end{tabular}

Nota. Las negritas indican las cargas factoriales mayores. Factor 1 = AFO de Oposición; Factor 2 = AFO de Esquí, Patinaje y Patinete; Factor $3=$ AFO de Resistencia No Intrusiva; Factor 4 = AFO de Gimnasio y Culturismo; Factor 5 = AFO de Ejercicios Aeróbicos; Factor 6 = AFO de Competición Individual; Factor 7 = AFO de Natación.

Tabla 2. Características Demográficas por Conglomerado en la AFO de Resistencia $(\mathrm{N}=329)$

\begin{tabular}{|c|c|c|c|c|c|c|c|c|}
\hline \multirow[b]{2}{*}{ Característica } & \multicolumn{4}{|c|}{$\begin{array}{l}\text { Conglomerado 1: AFO de Resistencia Baja-Normal } \\
\qquad(n=287)\end{array}$} & \multicolumn{4}{|c|}{$\begin{array}{l}\text { Conglomerado 2: AFO de Resistencia Excesiva } \\
\qquad(n=42)\end{array}$} \\
\hline & $\%$ & $M$ & $D T$ & $M d n$ & $\%$ & $M$ & $D T$ & $M d n$ \\
\hline Edad (años) & & 21,25 & 3,60 & 2,17 & & 21,01 & 2,68 & 20,17 \\
\hline Género (Mujer) & 55,7 & & & & 54,8 & & & \\
\hline Grado (Ciencias de los Deportes) & 39,0 & & & & 31,0 & & & \\
\hline Nivel educativo de la madre & & $1,75^{\mathrm{b}}$ & $0,95^{\mathrm{b}}$ & $2,00^{\mathrm{b}}$ & & 1,81 & 1,04 & 2,00 \\
\hline Nivel educativo del padre & & $1,82^{\mathrm{c}}$ & $0,99^{c}$ & $2,00^{c}$ & & 1,83 & 0,99 & 2,00 \\
\hline
\end{tabular}

Nota. ${ }^{\mathrm{a}} n=278 .{ }^{\mathrm{b}} n=285 .{ }^{\mathrm{c}} n=284$.

análisis de componentes principales (ACP; véase Apéndice A para una descripción detallada del análisis). La Tabla 1 muestra las cargas factoriales después de la rotación. En base a la literatura (Ford, 2007; García et al., 2011; Kondric et al., 2013; NIH, 2011), se identificaron los componentes como AFO de Oposición (Factor 1); AFO de Esquí, Patinaje y Patinete (Factor 2); AFO de Resistencia No Intrusiva ${ }^{3}$ (Factor 3); AFO de Gimnasio y Culturismo (Factor 4); AFO de Ejercicio aeróbico (Factor 5); AFO de Competición Individual (Factor 6); y AFO de Natación (Factor 7).

3 Con AFO de Resistencia No Intrusiva nos referimos a AF que generalmente se practican para mantener la condición física y se supone que interfieren mínimamente con la vida diaria.
A continuación, se llevó a cabo un análisis de conglomerados de k-medias con medida de la distancia euclidiana sobre las puntuaciones factoriales de la AFO de Resistencia No Intrusiva, para discriminar a un grupo de participantes que practicaban la AFO de resistencia en niveles muy altos o excesivos en comparación con el resto de la muestra. Denominamos los conglomerados AFO de Resistencia BajaNormal (conglomerado $1 ; \mathrm{n}=287$ ) y AFO de Resistencia Excesiva (Conglomerado 2; $\mathrm{n}=42$ ). Las características demográficas por conglomerado se presentan en la Tabla 2. En el Apéndice B, se explica el análisis con más detalle. La Tabla B.1 muestra las diferencias entre los conglomerados en la participación en cada AFO. 
Para la medición de los PARs no relacionados con drogas se utilizó el cuestionario MULTICAGE CAD-4 (Pedrero Pérez et al., 2007). El cuestionario consta de 32 ítems que evalúan los PARs relacionados con el alcohol, las drogas, videojuegos, sexo, alimentación, juego de azar, internet y gastar. Aquí, hemos utilizado las variables no relacionadas con drogas. Todos los ítems son dicotómicos (sí/no). En cada subescala PAR, ninguna o una respuesta afirmativa indican la ausencia de un problema clínico relevante, dos respuestas afirmativas indican la posible existencia de un problema, tres respuestas afirmativas indican un problema clínico muy probable y cuatro respuestas afirmativas, la existencia casi definitiva de un problema (una puntuación de 2 o más se considera clínicamente significativa). El cuestionario se aplica fácilmente y presenta adecuadas propiedades psicométricas y discriminativas (Pedrero Pérez et al., 2007).

Para contrastar la incidencia de la conducta alimentaria problemática en función de la participación en AFO, se identificaron individuos con puntuaciones PAR relacionadas con la alimentación por encima de un umbral clínico significativo: Dividimos las puntuaciones PAR relacionadas con la alimentación en $0-1$, que indica una probabilidad baja, y $3-4$, que indica una alta probabilidad de problemas clínicos. Los participantes que puntuaron 2 no se incluyeron para disminuir la posibilidad de falsos positivos.

La Impulsividad se evaluó con la versión en español de la Brief UPPS-P Impulsive Behavior Scale ([Escala Breve de Comportamiento Impulsivo]; Cándido, Orduña, Perales, Verdejo-García, y Billieux, 2012; Whiteside, Lynam, Miller, y Reynolds, 2005), basada en el modelo explicado anteriormente. Cada rasgo se mide con cuatro ítems, en una escala Likert de cuatro puntos que van desde 1 (muy de acuerdo) a 4 (totalmente en desacuerdo). Las propiedades psicométricas son satisfactorias y pueden encontrarse en Cándido et al. (2012).

\section{Análisis estadísticos}

Para la primera hipótesis sobre las relaciones entre la AFO y PARs no relacionados con drogas, se realizó un análisis de correlación parcial incluyendo los factores AFO, las puntuaciones PAR no-relacionados con drogas y los rasgos de personalidad impulsiva. Las variables demográficas (género, edad, años de educación y nivel de educación completado por la madre) se incluyeron en el análisis como variables de control. Estas variables ya se habían asociado con los PARs relacionados con drogas (Gardner, 1994; Lamptey, 2005) y en el presente estudio, correlacionaban o con la AFO o con las puntuaciones PAR no-relacionados con drogas. Si un participante estudiaba o no en la Facultad de Ciencias del Deporte también entró en el análisis como covariable (para una descripción detallada del análisis véase Apéndice C).

Para el segundo grupo de hipótesis se exploraron los potenciales correlatos clínicos de los diferentes patrones de AFO mostrados por los conglomerados de AFO de Resistencia Baja-Normal y AFO de Resistencia Excesiva. En primer lugar, mediante la prueba exacta de Fisher, se comparó la proporción de individuos que presentaron niveles clínicamente significativos entre el PAR relacionado con la alimentación entre los dos grupos AFO de resistencia. En segundo lugar, se analizaron las diferencias entre grupos por ítem del MULTICAGE CAD-4 en PAR relacionado con la alimentación, de nuevo con la prueba exacta de Fisher. Finalmente, se compararon los dos grupos en cada uno de los rasgos de personalidad impulsiva mediante de pruebas t de muestras independientes.

Realizamos todos los análisis con el IBM SPSS Statistics 20, y aplicamos un criterio de significación de 0,05.

\section{Resultados}

\section{Características de la muestra}

Las AFOs más populares, practicadas por al menos 15 estudiantes femeninos y masculinos de la facultad de Ciencias del Deporte así como de otros facultades, incluían correr $(36,4 \%$, incluyendo carrera lenta y carrera por senderos), fútbol $(23,7 \%)$, culturismo $(21,9 \%$, incluyendo el levantamiento de pesas), ejercicio aeróbico (17,9\%, incluyendo la expresión corporal, la danza, stepping, pilates, yoga, acrobacia, danza de poste, spinning, y AFOs similares guiadas), gimnasia en casa $(17,6 \%)$, ciclismo $(16,4 \%)$, gimnasia en el gimnasio (14,0\%), pádel $(13,7 \%)$, senderismo (13,7\%, incluyendo el trekking y el montañismo), natación $(8,5 \%)$, deportes de invierno $(7,6 \%)$, baloncesto $(7,3 \%)$, tenis $(7,0 \%)$, atletismo $(4,6 \%)$ y patinaje $(4,6 \%)$. Algunos participantes practicaban más de una actividad, mientras que otros $(\mathrm{n}=54)$ no practicaban ninguna. La media de tiempo de AFO fue $8,08 \mathrm{~h} / \mathrm{semana}(\mathrm{DT}=8,13$ ). La mediana de la muestra fue de 6 horas, y el $50 \%$ central de los encuestados pasaba entre 1,5 y 12 horas semanalmente realizando AFO. Las frecuencias, clasificadas por género y facultad, se muestran en la Tabla 3.

Tabla 3. AFO Practicada en Horas por Semana $(\mathrm{N}=329)$

\begin{tabular}{|c|c|c|c|c|c|c|c|c|c|c|c|c|}
\hline \multirow[b]{2}{*}{ Género } & \multicolumn{6}{|c|}{ Facultad de Ciencias del Deporte } & \multicolumn{6}{|c|}{ Otras facultades } \\
\hline & $n$ & $\%$ & $M$ & $D T$ & $M d n$ & $I Q R$ & $n$ & $\%$ & M & $D T$ & $M d n$ & $I Q R$ \\
\hline Mujer & 39 & 11,9 & 8,92 & 7,00 & 6,25 & $14,00-4,50$ & 144 & 43,8 & 4,73 & 5,45 & 3,00 & $7,19-0,06$ \\
\hline Varón & 86 & 26,1 & 12,63 & 9,02 & 11,21 & $16,00-6,38$ & 60 & 18,2 & 9,06 & 7,10 & 7,00 & $14,77-1,50$ \\
\hline
\end{tabular}


Tabla 4. Puntuaciones en PARs no relacionados con drogas del Cuestionario MULTICAGE CAD-4 $(\mathrm{N}=329)$

\begin{tabular}{|c|c|c|c|c|c|c|c|c|c|c|c|c|}
\hline \multirow{2}{*}{$\begin{array}{l}\text { Puntuación } \\
\text { PAR }\end{array}$} & \multicolumn{2}{|c|}{ Videojuegos } & \multicolumn{2}{|c|}{ Sexo } & \multicolumn{2}{|c|}{ Alimentación } & \multicolumn{2}{|c|}{ Juego de azar } & \multicolumn{2}{|c|}{ Internet } & \multicolumn{2}{|c|}{ Gasto } \\
\hline & $n$ & $\%$ & $n$ & $\%$ & $n$ & $\%$ & $n$ & $\%$ & $n$ & $\%$ & $n$ & $\%$ \\
\hline 0 & 281 & 85,4 & 267 & 81,2 & 191 & 58,1 & 311 & 94,5 & 124 & 37,7 & 246 & 74,8 \\
\hline 1 & 28 & 8,5 & 39 & 11,9 & 76 & 23,1 & 11 & 3,3 & 90 & 27,4 & 48 & 14,6 \\
\hline 2 & 15 & 4,6 & 16 & 4,9 & 31 & 9,4 & 4 & 1,2 & 64 & 19,5 & 23 & 7,0 \\
\hline 3 & 2 & 0,6 & 4 & 1,2 & 18 & 5,5 & 2 & 0,6 & 30 & 9,1 & 9 & 2,7 \\
\hline 4 & 2 & 0,6 & 2 & 0,6 & 12 & 3,6 & 0 & 0,0 & 20 & 6,1 & 2 & 0,6 \\
\hline Faltan datos & 1 & 0,3 & 1 & 0,3 & 1 & 0,3 & 1 & 0,3 & 1 & 0,3 & 1 & 0,3 \\
\hline$M(D T)$ & \multicolumn{2}{|c|}{$0,22(0,36)$} & \multicolumn{2}{|c|}{$0,28(0,67)$} & \multicolumn{2}{|c|}{$0,73(1,08)$} & \multicolumn{2}{|c|}{$0,08(0,36)$} & \multicolumn{2}{|c|}{$1,18(1,21)$} & \multicolumn{2}{|c|}{$0,39(0,79)$} \\
\hline
\end{tabular}

La Tabla 4 muestra la distribución de las frecuencias de PARs no relacionados con drogas en la muestra (según el cuestionario MULTICAGE CAD-4). Todas las distribuciones eran positivamente asimétricas: La mayoría de la muestra tenía bajas puntuaciones en las variables PAR no-relacionados con drogas. Como se mencionó anteriormente, una puntuación de 2 o superior indica la posible existencia de un PAR no relacionado con drogas. Como se ve por las medias y el número relativamente alto de puntuaciones de riesgo de 2 o más, bastantes encuestados mostraron potenciales PARs relacionados con la alimentación $(18,5 \%)$ y con internet $(34,7 \%)$, de los cuales una gran parte muy probablemente presenta esos problemas (puntuación de 3 o 4): respectivamente, el $9,1 \%$ y el $15,2 \%$.

Los rasgos de personalidad impulsiva medidos con la UPPS-P oscilan en un rango entre 1 (bajos rasgos de personalidad impulsiva) a 4 (altos rasgos de la personalidad impulsiva). Las puntuaciones medias fueron: urgencia negativa, $M=2,38(D T=0,74)$; urgencia positiva, $M=2,47$ $(D T=0,61)$; búsqueda de sensaciones, $M=2,58$ ( $D T=$ $0,75)$; falta de premeditación, $M=1,98(D T=0,56)$; y falta de perseverancia, $M=1,77(D T=0,59)$. La media general fue de 2,24 $(D T=0,42)$. Un participante no completó la escala.

\section{Relaciones entre las variables de interés}

Utilizamos correlaciones parciales para explorar las asociaciones entre las variables estudiadas. Los posibles factores de confusión que se describen en el Apéndice $\mathrm{C}$ (género, edad, estudiar o no en la facultad de Ciencias del Deporte, años de educación, y nivel educativo completado por la madre) se introdujeron en los análisis como variables control. Las correlaciones se muestran en la Tabla 5, con las asociaciones relevantes para las hipótesis en negrita. Una relación inversa entre las AFO y los PARs no relacionados con drogas sólo apareció para las AFO de Gimnasio y culturismo y los PARs relacionados con los videojuegos. Encontramos una relación positiva para el mismo factor de AFO de Gimnasio y Culturismo y los PARs relacionados con el sexo. Por último, como se predijo, una correlación positiva apareció para la AFO de Resistencia No Intrusiva y los PARs relacionados con la alimentación.

En estas asociaciones entre la AFO y los PARs no relacionados con drogas encontramos una posible implicación o efecto moderador de los rasgos de personalidad impulsiva sólo para la búsqueda de sensaciones en la relación positiva entre la AFO de Gimnasio y Culturismo y los PARs relacionados con el sexo. Como se muestra en Tabla 5, la búsqueda de sensaciones correlacionó tanto con la AFO de Gimnasio y Culturismo como con los PARs relacionados con el sexo.

\section{PARs relacionados con la alimentación en los practi- cantes de AFO de resistencia excesiva}

En el conglomerado de AFO de Resistencia Baja-Normal ( $\mathrm{n}=287$ ) un $7,7 \%$ mostró una puntuación de 3 o más en los PARs relacionadas con la alimentación, frente a un 19,0\% del conglomerado de AFO de Resistencia Excesiva $(\mathrm{n}=42)$. Un análisis de los datos con la prueba exacta de Fisher, en el que se excluyeron a los individuos que puntuaron 2 en la subescala de los PAR relacionados con la alimentación, como se explicó anteriormente, arrojó una asociación significativa entre el conglomerado de la AFO de resistencia y los PARs relacionados con la alimentación, $\mathrm{p}=0,017, \mathrm{OR}=3.10,95 \%$ CI $[1.26,7.63]$. Apoyando la hipótesis, la proporción de los encuestados que presenta puntuaciones por encima del umbral clínicamente significativo en los PARs relacionados con la alimentación (puntuación 3-4 en la subescala MULTICAGE en los trastornos de la alimentación, en contraposición a los individuos con puntuaciones de $0-1$ ) fue 2.64 veces mayor en el conglomerado de AFO de Resistencia Excesiva.

Las pruebas exactas de Fisher llevadas a cabo con toda la muestra $(\mathrm{N}=329)$ mostró que los grupos sólo difirieron significativamente en las respuestas afirmativas al primer ítem de la variable PARs relacionados con la alimentación, “Se ha provocado alguna vez el vómito para evitar ganar peso?", $\mathrm{p}=0,040, \mathrm{OR}=2.43,95 \%$ CI $[1.06,5.57]$. La proporción de individuos que respondió "sí" fue 2.12 veces mayor en el conglomerado de AFO de Resistencia Exce- 


\begin{tabular}{|c|c|c|c|c|c|c|c|c|c|c|c|c|c|c|c|c|c|c|}
\hline & 1 & 2 & 3 & 4 & 5 & 6 & 7 & 8 & 9 & 10 & 11 & 12 & 13 & 14 & 15 & 16 & 17 & 18 \\
\hline 1. AFO de Oposición & - & & & & & & & & & & & & & & & & & \\
\hline 2. AfO Esquí, Patinaje y Patinete &,- 07 & - & & & & & & & & & & & & & & & & \\
\hline 3. AFO de Resistencia No Intrusiva &, 02 &, 03 & - & & & & & & & & & & & & & & & \\
\hline 4. AFO de Gimnasio y Culturismo &,$- 13^{*}$ &,- 02 &, 01 & - & & & & & & & & & & & & & & \\
\hline 5. AFO de Ejercicios Aeróbicos &, $11^{\circ}$ &, 04 &, 00 &, 08 & - & & & & & & & & & & & & & \\
\hline 6. AFO de Competición Individual &,- 03 &, 00 & 01 &,- 02 &, 00 & - & & & & & & & & & & & & \\
\hline 7. AFO de Natación &, 01 &, $10^{\circ}$ &,- 01 &,- 01 &,- 02 &,- 02 & - & & & & & & & & & & & \\
\hline 8. PARs relacionados con videojuegos &, 01 &,- 02 &, 02 &,$- 13^{\star}$ &,- 06 & ,06 &, 02 & - & & & & & & & & & & \\
\hline 9. PARs relacionados con el sexo &,- 06 &, 06 &,- 02 &, $16 * *$ &,- 02 &,- 04 &,- 01 & 09 & - & & & & & & & & & \\
\hline 10. PARs relacionados con la alimentación &,- 06 &,- 01 &, $17^{\star *}$ &, 04 &, $10^{\circ}$ &, 07 &,- 02 &,- 01 &, 07 & - & & & & & & & & \\
\hline 11. PARs relacionados con juego de azar &, 05 &,- 09 &,- 03 &, 09 &, 06 &,- 01 &,- 03 &, 06 &, $18^{\star *}$ & 07 & - & & & & & & & \\
\hline 12. PARs relacionados con internet &, 03 &,- 03 &, 06 &, 04 &,- 04 &, 01 &,- 03 &, $16^{\star \star}$ &, $17^{\star \star}$ &, $17^{\star \star}$ &, 06 & - & & & & & & \\
\hline 13. PARs relacionados con el gasto &,- 02 &,- 01 &,- 01 &, 06 &, 00 &,- 06 &,- 03 &, $12^{\star}$ &, $32^{\star \star}$ &, $16^{\star \star}$ & ,06 &, $22^{\star \star}$ & - & & & & & \\
\hline 14. Urgencia negativa &,- 02 &, 00 &, 00 &, 03 &, 00 &, 02 &,- 07 & ,06 &, $19^{\star *}$ &, $21^{\star *}$ &, $10^{\circ}$ &, $15^{\star *}$ &, $18^{\star \star}$ & - & & & & \\
\hline 15. Urgencia positiva &,- 08 &, 08 &, 04 & ,09 &,- 01 &, 03 &,- 02 &, 03 &, $23^{\star \star}$ &, $15^{\star \star}$ &, $15^{\star}$ &, $15^{\star \star}$ &, $24^{\star \star}$ &, $50^{\star \star}$ & - & & & \\
\hline 16. Búsqueda de sensaciones &, 00 &, $17^{\star \star}$ &,- 01 &, $17^{\star \star}$ &,- 03 &, $17^{\star \star}$ &, 00 &,- 06 &, $26^{* *}$ & 07 &, $11^{\star}$ &, $11^{\circ}$ &, $12^{*}$ &, $19^{\star *}$ &, $41^{\star *}$ & - & & \\
\hline 17. Falta de premeditación &,- 05 & ,09 &,- 02 &, 07 &, 04 &,- 04 &, 02 & 07 &, $19^{\star *}$ &,- 01 &, 00 &, $11^{\circ}$ & 08 &, $23^{\star *}$ &, $33^{* *}$ &, $29^{\star *}$ & - & \\
\hline 18. Falta de perseverancia & 01 &,$- 12^{\star}$ &,- 05 &,- 04 &,- 04 &,- 04 &,- 05 &, $12^{*}$ &, $15^{* *}$ & 05 &, $20^{\star *}$ &, $21^{\star *}$ &, $18^{\star \star}$ &, $11^{\circ}$ &, $25^{\star *}$ & ,05 &, $27^{\star \star}$ & - \\
\hline
\end{tabular}

Nota. La negrita indica las mayores correlaciones relacionadas con las hipótesis. Variables de control fueron edad, género, estudiar o no en la facultad de Ciencias del Deporte, años de educación, y nivel educativo completado por la madre. ${ }^{\circ} p<0,10 .{ }^{\star} p<0,05 .{ }^{\star \star} p<0,01$.

siva. Confirmando las correlaciones parciales previamente mostradas (Tabla 5), las pruebas t no mostraron diferencias significativas entre los conglomerado con respecto a los rasgos de personalidad impulsiva: El valor de p más bajo fue de $p=0,201$.

\section{Discusión}

Resumiendo los principales resultados, los datos revelan un número muy limitado de asociaciones entre la AFO y PARs no relacionados con drogas y las adicciones conductuales en una muestra comunitaria de estudiantes universitarios. Cuanto más altos eran los niveles de práctica de AFO de Gimnasio y Culturismo, menos PARs relacionados con videojuegos y más PARs relacionados con el sexo se registraron. La alta participación en las AFOs de resistencia se asoció a más PARs relacionados con la alimentación, específicamente con provocarse el vómito como una estrategia para el control de peso. Además, una cantidad considerable de relaciones positivas apoyan la hipotética asociación entre los rasgos de personalidad impulsiva y los PARs no relacionados con drogas. Sin embargo, no se encontraron claras asociaciones entre los rasgos de personalidad impulsiva y las AFOs que a su vez, podrían ser responsables de una relación entre las AFOs y los PARs no relacionados con drogas.

El modelo de la fuerza del autocontrol (Baumeister et al., 2011; Baumeister et al., 2007) predice relaciones inversas entre la participación en la AFO y PARs no relacionados con drogas. Parcialmente de acuerdo con este modelo, encontramos que niveles más bajos de participación en la AFO de Gimnasio y Culturismo correlacionan con niveles más altos de PARs relacionadas con videojuegos. Sin embargo, si bien el modelo propone que la disponibilidad de recursos de autocontrol es el núcleo de los PARs y de la implicación regular en actividades de esfuerzo (Dorris et al., 2012; Joseph et al., 2011), en nuestro estudio no se encontró ninguna indicación de rasgos de personalidad impulsiva que pudieran ser responsables de la asociación. Por otra parte, 
la hipótesis de desplazamiento podría explicar la asociación inversa entre la AFO de Gimnasio y Culturismo y los PARs relacionadas con videojuegos. Esa propuesta establece que el tiempo dedicado a los comportamientos sedentarios desplaza el tiempo que se podría dedicar a AF (Ballard, Gray, Reilly, y Noggle, 2009; Mansoubi, Pearson, Biddle, y Clemes, 2014). Consistentemente con nuestros resultados, Ballard y colegas (2009) encontraron una relación negativa entre el tiempo dedicado a videojuegos y los niveles de AF.

El factor de AFO de Gimnasio y Culturismo estaba positivamente relacionado con los PARs sexuales, y la alta búsqueda de sensaciones correlacionaba simultáneamente con ambos constructos. Hasta donde sabemos, la relación entre AF y una excesiva conducta sexual nunca se ha analizado directamente. Sin embargo, investigaciones previas sugieren (1) una asociación entre Culturismo (Litt y Dodge, 2008; McCreary y Sasse, 2000), incluso con su exceso (Hale, Roth, DeLong, y Briggs, 2010; Hurst, Hale, Smith, y Collins, 2000) y el impulso de musculación, especialmente entre los varones (McCreary, 2012; McCreary et al., 2000); (2) asociaciones entre la musculatura (en hombres) y la delgadez (en mujeres) con ideales del atractivo (Murnen y Don, 2012; Murray, Rieger, Touyz, y De la Garza García, 2010); y (3) una asociación entre la motivación por el atractivo y la frecuencia de las relaciones sexuales (Brody, 2004; Filiault, 2007; Swami, Diwell, y McCreary, 2014). Nuestros resultados sugieren la posibilidad de que la práctica de la AFO de Gimnasio y Culturismo puede asociarse con no sólo una actividad sexual más intensa, sino también con sus consecuencias negativas en forma de síntomas de PARs de hipersexualidad.

Además del impulso hacia el atractivo, una explicación alternativa (no necesariamente incompatible) incluye la existencia de un factor común de la personalidad asociado con ambos comportamientos. En concordancia con esa posibilidad, la búsqueda de sensaciones correlaciona tanto con la AFO de Gimnasio y Culturismo como con los PARs relacionados con el sexo. Se han encontrado niveles más altos de búsqueda de sensaciones entre los miembros de los clubes de Gimnasio (Lichtenstein et al., 2014), y la búsqueda de sensaciones se ha revelado como un robusto predictor de la conducta sexual de riesgo (e.g., Hoyle, Fejfar, y Miller, 2000; Zapolski, Cyders, y Smith, 2009).

La dependencia primaria de AF no se confirmó: No se encontraron marcadores de rasgo de personalidad impulsiva (se esperaba que fuesen principalmente urgencia negativa y búsqueda de sensaciones; Billieux et al., 2007; Dick et al., 2010; Navas, Torres, Cándido, y Perales, 2014; Navas, Torres, Vilar, et al., 2014) que potencialmente subyaciesen a las asociaciones positivas entre medidas de AFO de resistencia excesiva y PARs no relacionados con drogas. Sin embargo, en concordancia con la hipótesis de dependencia secundaria a la AF (de Coverley Veale, 1987), las puntuaciones altas en el factor de AFO de Resistencia No Intrusiva (que incluye correr, gimnasia en casa y senderismo) correlacionaban con signos de PARs relacionados con la alimentación. Además, en un conglomerado de individuos que mostraban patrones muy elevados de práctica de esto tipo de AFO (etiquetados como AFO de Resistencia Excesiva) se duplicó con creces la probabilidad de presentar síntomas de PARs relacionados con la alimentación por encima del umbral clínicamente significativo, en comparación con los participantes de AFO de Resistencia Baja-Normal. Esto es congruente con investigaciones previas, lo que indica que la participación excesiva en $\mathrm{AF}$ de resistencia y los patrones de alimentación desordenados a menudo van de la mano (Allegre et al., 2006; Grandi et al., 2011; Lichtenstein et al., 2014). Además, como resultado novedoso, encontramos que usar el vómito como una estrategia de control de peso distingue entre los participantes en AFO de Resistencia Baja-Normal y los participantes en AFO de Resistencia Excesiva.

El rasgo de urgencia negativa de la personalidad impulsiva correlacionaba con los PARs relacionados con la alimentación. Este resultado es consistente con los informes de urgencia negativa como un fuerte predictor de psicopatología, especialmente en el campo de los PARs (Billieux et al., 2007; Dick et al., 2010). No encontramos la asociación potencial entre los PARs relacionados con la alimentación y la búsqueda de sensaciones propuesta por investigaciones previas como una señal de exposición temprana a sustancias potencialmente adictivas (e.g.,Cyders, Flory, Rainer, y Smith, 2009), ni con el comer en exceso y la obesidad (e.g., Schag, Schönleber, Teufel, Zipfel, y Giel, 2013). Esto sugiere que el tipo de PARs relacionados con la alimentación en la muestra actual que estaba relacionado con AFO excesivo no está relacionado con la adicción a la comida (e.g., Lerma-Cabrera, Carvajal, y Lopez-Legarrea, 2016), sino que está más estrechamente relacionado con formas clínicas y subclínicas de anorexia y bulimia. La relación entre la AFO excesiva y el vómito también apuntan en esa dirección.

\section{Fortalezas y Limitaciones}

Hay un número de limitaciones que potencialmente afectan a las conclusiones presentadas. Primero, se requieren investigaciones longitudinales con el fin de examinar las direcciones de los resultados correlacionales en nuestro estudio trasversal de autoinforme. Otra limitación es el hecho de que, dada la naturaleza exploratoria del estudio, algunas hipótesis eran bastante abiertas. Dado que no utilizamos ningún método para el ajuste del alfa, esto podría considerarse como fishing. Aún así, esto no se aplica a la asociación encontrada entre la AFO de resistencia y los PARs relacionados con la alimentación, que esperábamos sobre la base de una hipótesis mucho más específica.

Además, aunque parte de nuestras hipótesis estaban basadas en los modelos de dependecia a la AF (e.g., de Coverley Veale, 1987), en realidad, para AF, las características de adicción no se han medido: Las medidas puramente conductua- 
les no se asocian necesariamente a los síntomas de dependencia a la AF (Adkins y Keel, 2005; Mond, Hay, Rodgers, y Owen, 2006). Una fortaleza relevante es la adaptación del GAFQ (Armstrong et al., 2006) sin alterar su sistema de medición, que nos permitió evaluar distintas modalidades de deportes: La importancia de los tipos de deporte en relación con cuestiones de autorregulación había recibido muy poca atención en investigaciones anteriores.

La generalización de los resultados se apoya en el hecho de que las AFOs más practicadas son comparables a los deportes más practicados en España (García et al., 2011). Además, en un estudio con una población de todas las edades (Rodríguez Monje, Pedrero Pérez, Fernández Girón, Gallardo Alonso, y Sanz Cuesta, 2009), se encontraron distribuciones muy similares-muy asimétricas-de PARs no relacionados con drogas. En nuestro estudio, un tamaño mayor de la muestra podría haber permitido más participantes con puntuaciones de problemas potencialmente clínicos y, de esta manera, hubiera permitido una mayor potencia estadística. Además, el cuestionario puede ser demasiado sensible respecto del abuso de internet (Billieux, Schimmenti, Khazaal, Maurage, y Heeren, 2015), en el cual un alto porcentaje de la muestra puntuó por encima del umbral de riesgo para problemas clínicos. No obstante, los índices de prevalencia más plausibles encontrados para otros PARs no relacionados con drogas, también muestran porcentajes considerables de conductas adictivas potencialmente clínicas entre universitarios. Esto apoya la relevancia del reconocimiento e intervención tempranos, sobre todo porque las personas que realizan comportamientos adictivos a menudo experimentan síntomas psicopatológicos comórbidos e incluso ideación suicida (Bousoño et al., 2017; Martín-Fernández et al., 2017). Evaluar correctamente los perfiles individuales de comportamientos problemáticos potencialmente clínicos, como los PARs no relacionados con drogas y los patrones de práctica de AFO, ayuda a identificar a las personas en riesgo, contribuye a la prevención, y ayuda a establecer planes de tratamiento adaptados al perfil de una persona (Martín-Fernández et al., 2017). El estudio tiene grandes fortalezas con respecto a los PARs relacionados con la alimentación. La probabilidad de que hayamos detectado realmente patología autorregulatoria relacionada con la alimentación es alta, a la vista de numerosos indicadores. La urgencia negativa se asoció con los PARs relacionados con la alimentación, y hay evidencia substancial que ha mostrado que este rasgo de personalidad impulsiva es un indicador de patología clínica (Billieux et al., 2007; Dick et al., 2010). Además, Pedrero Pérez et al. (2007) encontraron una sensibilidad diagnóstica para el punto de corte de dos o más respuestas afirmativas, que indica un problema clínico por encima del $90 \%$ de abuso de sustancias: En el presente estudio, se utilizó un punto de corte más conservador de tres o cuatro respuestas afirmativas para indicar PARs clínicos relacionados con la alimentación. Por último, la diferencia en los PARs relacionados con la alimentación entre participantes de AFO de Resistencia Baja-Normal y participantes de AFO de Resistencia Excesiva parece ser especialmente cierto para el uso del vómito como estrategia para evitar el aumento de peso: Se encontró previamente que este ítem del cuestionario MULTICAGE CAD-4 se relacionaba con ser diagnosticada de anorexia y bulimia (Rodríguez Monje et al., 2009), por lo tanto podría considerarse como un signo de patología clínica de la alimentación.

Por último, las comparaciones entre conglomerados relativos a la dimensión de AFO de resistencia sugieren fuertemente que la asociaciones entre AFO y PARs ocurren en niveles muy elevados (excesivos o clínicamente significativos) de ambos constructos. Esto puede ser una explicación para los pequeños efectos entre las variables continuas cuando se mide todo el rango, y requiere un nuevo examen.

\section{Conclusiones}

Con el fin de desarrollar planes de intervención y tratamiento eficaces para los PARs y las adicciones conductuales, la comprensión de la población en riesgo es imprescindible. El presente estudio ha ayudado a identificar los patrones de práctica de AFO y los rasgos de personalidad impulsiva como factores de riesgo y de protección para los PARs no relacionados con drogas, con el valor añadido de las exploraciones por modalidad de AFO. Los principales resultados novedosos fueron la relación inversa entre la AFO de Gimnasio y Culturismo y los PARs relacionadas con videojuegos; un triángulo de asociaciones positivas entre la AFO de Gimnasio y Culturismo, los PARs relacionados con el sexo y la búsqueda de sensaciones. Y, más importante, una relación positiva entre correr, gimnasia en casa, senderismo y las AFOs similares de resistencia con los PARs relacionados con la alimentación, con la aparición más frecuente del síntoma de vómitos del trastorno de la alimentación en personas que presentan una participación muy elevada en esas actividades. Esto resulta de particular importancia, puesto que el uso del vómito como una estrategia para perder peso es altamente indicativo de patología de la alimentación.

Los resultados de nuestro estudio sugieren una prevalencia sustancial de PARs no relacionados con drogas potencialmente clínicos y de adicciones conductuales entre los estudiantes universitarios, lo que muestra que es relevante reconocerlos, intervenir en ellos o prevenirlos. Por ello, se recomienda que futuras investigaciones bien diseñadas extiendan la evidencia para el rol de las AFOs como una conducta de riesgo y una estrategia de intervención no farmacéutica para los problemas de autorregulación en varias poblaciones, y además, que detecten los antecedentes psicológicos de esas asociaciones. Esto puede ayudar en la aclaración adicional de señales de alarma y posibilidades de tratamiento para comportamientos de riesgo y poco saludables con respecto a la autorregulación. 


\section{Reconocimientos}

Los autores quisieran agradecer a los estudiantes que han participado, así como a Harvey Owen, Ebel Floridia, Batsheva Mannheim y María Sherwood Smith por sus valiosas aportaciones al manuscrito.

La investigación que se describe en este manuscrito ha sido financiado por una subvención para el último autor, JCP, del Gobierno Español (Ministerio de Economía y Competitividad, Secretaría de Estado de Investigación, Desarrollo e Innovación; Convocatoria 2013 de Proyectos I+D de Excelencia), con número de referencia PSI2013-45055-P.

\section{Conflictos de interés}

Los autores declaran que no hay intereses financieros competidores.

\section{Referencias}

Adams, J. y Kirkby, R. (2001). Exercise dependence and overtraining: The physiological and psychological consequences of excessive exercise. Sports Medicine Training and Rehabilitation, 10, 199-222. doi:10.1080/10578310210395.

Adkins, E. C. y Keel, P. K. (2005). Does "excessive" or "compulsive" best describe exercise as a symptom of bulimia nervosa? The International Journal of Eating Disorders, 38, 24-29. doi:10.1002/eat.20140.

Allegre, B., Souville, M., Therme, P. y Griffiths, M. (2006). Definitions and measures of exercise dependence. Addiction Research $\mathcal{E}$ Theory, 14, 631-646. doi:10.1080/16066350600903302.

American Psychiatric Association. (2013). Diagnostic and statistical manual of mental disorders (5th ed.). Arlington, VA: American Psychiatric Publishing.

Armstrong, T. y Bull, F. (2006) Development of the World Health Organization Global Physical Activity Questionnaire (GPAQ). Journal of Public Health, 14, 66-70. doi:10.1007/s10389-006-0024-x.

Ballard, M., Gray, M., Reilly, J. y Noggle, M. (2009). Correlates of video game screen time among males: Body mass, PA, and other media use. Eating Behaviors, 10, 161167. doi:10.1016/j.eatbeh.2009.05.001.

Baumeister, R. F. y Tierney, J. (2011). Willpower: Rediscovering the Greatest Human Strength. New York, NY: The Penguin Press.

Baumeister, R. F., Vohs, K. D. y Tice, D. M. (2007). The strength model of self-control. Current Directions in Psychological Science, 16, 351-355. doi:10.1111/j.14678721.2007.00534.x.

Billieux, J., Gay, P., Rochat, L. y Van der Linden, M. (2010). The role of urgency and its underlying psychological mechanisms in problematic behaviours. Behavioural
Research and Therapy, 48, 1085-1096. doi:10.1016/j. brat.2010.07.008.

Billieux, J., Schimmenti, A., Khazaal, Y., Maurage, P. y Heeren, A. (2015). Are we overpathologizing everyday life? A tenable blueprint for behavioral addiction research. Journal of Behavioral Addictions, 4, 119-123. doi:10.1556/2006.4.2015.009.

Billieux, J., Van der Linden, M. y Ceschi, G. (2007). Which dimensions of impulsivity are related to cigarette craving? Addictive Behaviors, 32, 1189-1199. doi:10.1016/j. addbeh.2006.08.007.

Bousoño, M., Al-Halabí, S., Burón, P., Garrido, M., Díaz-Mesa, E. M., Galván, G.,... Bobes, J. (2017). Substance use or abuse, internet use, psychopathology and suicidal ideation in adolescents. Adicciones, 29, 97-104. doi:10.20882/adicciones.811.

Bratland-Sanda, S., Martinsen, E. W., Rosenvinge, J. H., Rø, $\varnothing .$, Hoffart, A. y Sundgot-Borgen, J. (2011). Exercise dependence score in patients with longstanding eating disorders and controls: The importance of affect regulation and physical activity intensity. European Eating Disorders Review, 19, 249-255. doi:10.1002/erv.971.

Breuer, S. y Kleinert, J. (2009). Primäre Sportsucht und bewegungsbezogene Abhängigkeit - Beschreibung, Erklärung und Diagnostik. In D. Batthyany y A. Pritz (Eds.), Rausch ohne Drogen. Substanzungebundene Süchte (pp. 191-218). Vienna, Austria: Springer.

Brody, S. (2004). Slimness is associated with greater intercourse and lesser masturbation frequency. Journal of Sex and Marital Therapy, 30, 251-261. doi:10.1080/00926230490422368.

Bull, F. C., Maslin T. S. y Armstrong, T. (2009). Global Physical Activity Questionnaire (GPAQ): Nine country reliability and validity study. Journal of Physical Activity and Health, 6, 790-804. doi:10.1123/jpah.6.6.790.

Cándido, A., Orduña, E., Perales, J. C., Verdejo-García, A. y Billieux, J. (2012). Validation of a short Spanish version of the UPPS-P impulsive behaviour scale. Trastornos Adictivos, 14, 73-78. doi:10.1016/S15750973(12) 70048-X.

Cyders, M. A., Flory, K., Rainer, S. y Smith, G. T. (2009). The role of personality dispositions to risky behavior in predicting first-year college drinking. Addiction, 104, 193-202. doi:10.1111/j.1360-0443.2008.02434.x.

Cyders, M. A., Smith, G. T., Spillane, N. S., Nichea, S., Fischer, S., Annus, A. M. y Peterson, C., (2007). Integration of impulsivity and positive mood to predict risky behavior: Development and validation of a measure of positive urgency. Psychological Assessment, 19, 107-118. doi:10.1037/1040-3590.19.1.107.

de Coverley Veale, D. M. W. (1987). Exercise dependence. British Journal of Addiction, 82, 735-740. doi:10.1111/j.1360-0443.1987.tb01539.x. 
Dick, D. M., Smith, G., Olausson, P., Mitchell, S. H., Leeman, R. F., O’Malley, S. S. y Sher, K. (2010). Understanding the construct of impulsivity and its relationship to alcohol use disorders. Addiction Biology, 15, 217-226. doi:10.1111/j.1369-1600.2009.00190.x.

Dorris, D. C., Power, D. A. y Kenefick, E. (2012). Investigating the effects of ego depletion on physical exercise routines of athletes. Psychology of Sport and Exercise, 13, 118-125. doi:10.1016/j.psychsport.2011.10.004.

Evenden, J. (1999). Impulsivity: A discussion of clinical and experimental findings. Journal of Psychopharmacology, 13, 180-192. doi:10.1177/026988119901300211.

Filiault, S. M. (2007). Measuring up in the bedroom: Muscle, thinness, and men's sex lives. International Journal of Men's Health, 6, 127-142. doi:10.3149/jmh.0602.127.

Ford, J. A. (2007). Substance use among college athletes: A comparison based on sport/team affiliation. Journal of American College Health, 55, 367-373. doi:10.3200/ JACH.55.6.367-373.

García, F. M. y Llopis, G. R. (2011). Encuesta sobre los hábitos deportivos en España 2010. Ideal democrático y bienestar personal. Madrid, Spain: Consejo Superior de Deportes, Centro de Investigaciones Sociológicas.

Grandi, S., Clementi, C., Guidi, J., Benassi, M. y Tossani, E. (2011). Personality characteristics and psychological distress associated with primary exercise dependence: An exploratory study. Psychiatry Research, 189, 270-275. doi:10.1016/j.psychres.2011.02.025.

Grant, J. E., Potenza, M. N., Weinstein, A. y Gorelick, D. (2010). Introduction to behavioral addictions. The American Journal of Drug and Alcohol Abuse, 36, 233-241. doi:1 0.3109/00952990.2010.491884.

Hale, B. D., Roth, A. D., DeLong, R. E. y Briggs, M. S. (2010). Exercise dependence and the drive for muscularity in male bodybuilders, power lifters, and fitness lifters. Body Image, 7, 234-239. doi:10.1016/j.bodyim.2010.02.001.

Hoyle, R. H., Fejfar, M. C. y Miller, J. D. (2000). Personality and sexual risk taking: A quantitative review. Journal of Personality, 68, 1203-1231. doi:10.1111/14676494.00132.

Hurst, R., Hale, B. D., Smith, D. y Collins (2000). Exercise dependence, social physique anxiety, and social support in experienced and inexperienced bodybuilders and weight lifters. British Journal of Sports Medicine, 11, 319-325. doi:10.1136/bjsm.34.6.431.

Joseph, R. J., Alonso-Alonso, M., Bond, D. S., Pascual-Leone, A. y Blackburn, G. L. (2011). The neurocognitive connection between physical activity and eating behaviour. Obesity Reviews, 12, 800-812. doi:10.1111/j.1467789X.2011.00893.x.

Knezevic-Budisin, B., Pedden, V., White, A., Miller, C. J. y Hoacken, P. N. S. (2015). A multifactorial conceptualization of impulsivity: Implications for research and clini- cal practice. Journal of Individual Differences, 36, 191-198. doi:10.1027/1614-0001/a000173.

Kondric, M., Sekulic, D., Petroczi, A., Ostojic, L., Rodek, L. y Ostojic, Z. (2011). Is there a danger for myopia in anti-doping education? Comparative analysis of substance use and misuse in Olympic racket sports calls for a broader approach. Substance Abuse Treatment, Prevention, and Policy, 6, 27. doi:10.1186/1747-597X-6-27.

Kondric, M., Sindik, J., Furjan-Mandic, G. y Schiefler, B. (2013). Participation motivation and student's physical activity among sport students in three countries. Journal of Sports Science and Medicine, 12, 10-18.

Lamptey, J.J. (2005). Socio-demographic characteristics of substance abusers admitted to a private specialist clinic. Ghana Medical Journal, 39, 2-7. doi:10.4314/gmj.v39i1.35973.

Landolfi, E. (2013). Exercise addiction. Sports Medicine, 43, 111-119. doi:10.1007/s40279-012-0013-x.

Lejoyeux, M., Tassain, V., Solomon, J. y Adès, J. (1997). Study of compulsive buying in depressed patients. Journal of Clinical Psychiatry, 58, 169-173. doi:10.4088/JCP.v58n0406.

Lerma-Cabrera, J. M., Carvajal, F. y Lopez-Legarrea, P. (2015). Food addiction as a new piece of the obesity framework. Nutrition Journal, 15, 5-10. doi:10.1186/ s12937-016-0124-6.

Lichtenstein, M. B., Christiansen, E., Elklit, A., Bilenberg, N. y Støving, R. K. (2014). Exercise addiction: A study of eating disorder symptoms, quality of life, personality traits and attachment styles. Psychiatry Research, 215, 410-416. doi:10.1016/j.psychres.2013.11.010.

Lisha, N. E. y Sussman, S. (2010). Relationship of high school and college sports participation with alcohol, tobacco, and illicit drug use: A review. Addictive Behaviors. 35, 399-407. doi:10.1016/j.addbeh.2009.12.032.

Litt, D. y Dodge, T. (2008). A longitudinal investigation of the Drive for Muscularity Scale: Predicting the use of performance enhancing substances and weightlifting among males. Body Image, 5, 346-351. doi:10.1016/j. bodyim.2008.04.002.

MacCallum, R. C., Widaman, K. F., Zhang, S. y Hong, S. (1999). Sample size in factor analysis. Psychological Methods, 4, 84-99. doi:10.1037/1082-989X.4.1.84.

Mansoubi, M., Pearson, N., Biddle, J. H. y Clemes, S. (2014). The relationship between sedentary behaviour and physical activity in adults: A systematic review. Preventive Medicine, 69, 28-35. doi:10.1016/j.ypmed.2014.08.028.

Martín-Fernández, M., Matalí, J. L., García-Sánchez, S., Pardo, M., Lleras, M. y Castellano-Tejedor, C. (2017). Adolescents with Internet Gaming Disorder (IGD): profiles and treatment response. Adicciones, 29, 125-133. doi:10.20882/adicciones.890.

McCreary, D. R. (2012). Muscularity and body image. In T. F. Cash (Ed.), Encyclopedia of body image and human appearance (pp. 561-567). San Diego, CA: Academic Press. 
McCreary, D. R. y Sasse, D. K. (2000). An exploration of the drive for muscularity in adolescent boys and girls. Journal of American College Health, 48, 297-304. doi:10.1080/07448480009596271.

Mond, J. M., Hay, P. J., Rodgers, B. y Owen, C. (2006). An update on the definition of "excessive exercise" in eating disorders research. The International Journal of Eating Disorders, 39, 147-153. doi:10.1002/eat.20214.

Murnen, S. K. y Don, B. P. (2012). Body image and gender roles. In T. F. Cash (Ed.), Encyclopedia of body image and human appearance. (pp. 128-134). San Diego, CA: Academic Press.

Murray, S. B., Rieger, E., Touyz, S. W. y De la Garza García (2010). Muscle dysmorphia and the DSM-V conundrum: Where does it belong? A review paper. International Journal of Eating Disorders, 43, 483-491. doi:10.1002/ eat.20828.

Navas, F. J., Torres, A., Cándido, A. y Perales, J. C. (2014). ¿'Nada' o 'un poco'? ¿'Mucho' o 'demasiado'?” La impulsividad como marcador de gravedad en niveles problemático y no problemático de uso de alcohol e Internet. Adicciones, 26, 159-167. doi:10.20882/adicciones.19.

Navas, J. F., Torres, A., Vilar, R., Verdejo-García, A., Catena, A. y Perales, J. C. (2014). Nonmonetary decision-making indices discriminate between different behavioral components of gambling. Journal of Gambling Studies, 31, 1545-1560. doi:10.1007/s10899-014-9482-1.

Pedrero Pérez, E. J., Rodríguez Monje, M. T., Gallardo Alonso, F., Fernández Girón, M., Pérez López, M. y Chicharro Romero, Y. J. (2007). Validación de un instrumento para la detección de trastornos de control de impulsos y adicciones: el MULTICAGE CAD-4. Trastornos Adictivos, 9, 269-278. doi:10.1016/S1575-0973(07)75656-8.

Perry, J. L. y Carroll, M. E. (2008). The role of impulsive behavior in drug abuse. Psychopharmacology, 200, 1-26. doi:10.1007/s00213-008-1173-0.

Petry, N. M., Stinson, F. S. y Grant, B. F. (2005). Comorbidity of DSM-IV pathological gambling and other psychiatric disorders: Results from the national epidemiologic survey on alcohol and related conditions. The Journal of Clinical Psychiatry, 66, 564-574. doi:10.4088/ JCP.v66n0504.

Raymond, N. C., Coleman, E. y Miner, M. H. (2003). Psychiatric comorbidity and compulsive/impulsive traits in compulsive sexual behavior. Comprehensive Psychiatry, 44, 370-380. doi:10.1016/S0010-440X(03)00110-X.

Rodríguez Monje, M. T., Pedrero Pérez, E. J., Fernández Girón, M., Gallardo Alonso, F. y Sanz Cuesta, T. (2009). Detección precoz de conductas adictivas en atención primaria: Utilización del MULTICAGE CAD-4. Atención Primaria, 41, 25-32. doi:10.1016/j.aprim.2008.04.004.

Schag, K., Schoenleber, J., Teufel, M., Zipfel, S. y Giel, K. E. (2013). Food-related impulsivity in obesity and binge eating disorder. A systematic review. Obesity Reviews, 14, 477-495. doi:10.1111/obr.12017.

Swami, V., Diwell, R. y McCreary, D. R. (2014). Sexuality and the drive for muscularity: Evidence of associations among British men. Body Image, 11, 542-546. doi:10.1016/j.bodyim.2014.08.008.

Verdejo-García, A., Lawrence, A. J. y Clark, L. (2008). Impulsivity as a vulnerability marker for substance-use disorders: Review of findings from high-risk research, problem gamblers and genetic association studies. Neuroscience Biobehavior Review, 32, 777-810. doi:10.1016/j. neubiorev.2007.11.003.

Vohs, K. D. y Baumeister, R. F. (Eds.). (2011). Handbook of self-regulation: Research, Theory, and Applications (2nd ed.). New York, NY: Guilford Press.

Whiteside, S. P. y Lynam, D. R. (2001). The five factor model and impulsivity: Using a structural model of personality to understand impulsivity. Personality and Individual Differences, 30, 669-689. doi:10.1016/S01918869(00)00064-7.

Whiteside, S. P., Lynam, D. R., Miller, J. D. y Reynolds, S. K. (2005). Validation of the UPPS impulsive behaviour scale: A four-factor model of impulsivity. European Journal of Personality, 19, 559-574. doi:10.1002/per.556.

Wills, T. A. y Dishion, T. J. (2004). Temperament and adolescent substance use: A transactional analysis of emerging self-control. Journal of Clinical Child and Adolescent Psychology, 33, 69-81. doi:10.1207/S15374424JCCP3301_7.

Wills, T. A., Isasi, C. R., Don Mendoza, M. A. y Ainette, M. G. M. A. (2007). Self-control constructs related to measures of dietary intake and physical activity in adolescents. Journal of Adolescent Health, 41, 551-558. doi:10.1016/j. jadohealth.2007.06.013.

Yen, J. Y., Ko, C. H., Yen, C. F., Chen, C. S. y Chen, C. C. (2009). The association between harmful alcohol use and Internet addiction among college students: Comparison of personality. Psychiatry and Clinical Neurosciences, 63, 218-224. doi:10.1111/j.1440-1819.2009.01943.x.

Zapolski, T. C. B., Cyders, M. A. y Smith, G. T. (2009). Positive urgency predicts illegal drug use and risky sexual behavior. Psychology of Addictive Behaviors, 23, 348-354. doi:10.1037/a0014684.

Ziemainz, H., Stoll, O., Drescher, A., Erath, R., Schipfer, M. y Zeulner, B. (2013). Die gefährdung zur sportsucht in ausdauersportarten. Deutsche Zeitschrift für Sportmedizin, 64, 57-64. doi:10.5960/dzsm.2012.057. 


\section{Apéndice A \\ Análisis factorial para las Actividades \\ Físicas de Ocio (AFO)}

Con el fin de reducir el número de AFOs evaluadas con el cuestionario CAFD-T a un conjunto manejable de dimensiones, se extrajo la combinación de dimensiones que mejor explicaba las correlaciones entre las diferentes AFOs con un análisis de componentes principales (ACP). Dado que las puntuaciones en las variables estudiadas difieren significativamente según el género y la facultad, sólo incluimos las actividades practicadas por ambos sexos, en la facultad de Ciencias del Deporte así como en otras facultades. Estos eran correr, fútbol, musculación, ejercicio aeróbico, gimnasia en casa, ciclismo, gimnasia en el gimnasio, pádel, senderismo, natación, deportes de invierno, baloncesto, tenis, atletismo y patinaje. La cantidad mínima de casos para hacer análisis factorial se cumplió (MacCallum, Widaman, Zhang, y Hong, 1999), con al menos 15 participantes en cada categoría de AFO. Analizamos las intercorrelaciones entre las variables, mostrando que todos los ítems correlacionaban significativamente con valores de 0,14-0,33 (una cola) por lo menos con otro ítem. La única excepción fue el baloncesto, si bien los datos mostraron una tendencia a la significación con el ejercicio aeróbico, $r=-0,07, p=0,116$. Esto indicaba una factorabilidad razonable y la ausencia de multicolinearidad. El test de esfericidad de Bartlett fue significativo, $\chi^{2}(105)=283,17, p$ $<0,001$, y todas las comunalidades (excepto el baloncesto, 0,43 ) estaban por encima de 0,50 , con una comunalidad promedia de 0,61. Esto confirmó que cada ítem compartía varianza con otras variables, tal que las relaciones entre las variables eran suficientemente grandes para hacer ACP. Teniendo en cuenta estos indicadores, se realizó el análisis factorial con los 15 elementos.

El análisis dio lugar a siete componentes, con autovalores mayores que el criterio de Kaiser de 1. En combinación, los componentes explicaron el $60,7 \%$ de la varianza. Había poca diferencia entre las soluciones Varimax y Oblimin: Para el modelo final elegimos la rotación Varimax. La Tabla 1 muestra las cargas factoriales después de la rotación. En base a la literatura (Ford, 2007; García et al., 2011; Kondric et al., 2013; NIH, 2011) identificamos los componentes como AFO de Oposición (Factor 1); AFO de Esquí, Patinaje y Patinete (Factor 2); AFO de Resistencia No Intrusiva (Factor 3); AFO de Gimnasio y Culturismo (Factor 4); AFO de Ejercicio Aeróbico (Factor 5); AFO de Competición Individual (Factor 6); y AFO de Natación (Factor 7). 


\section{Apéndice B \\ Análisis de conglomerados para la AFO de Resistencia}

Posteriormente, se llevó a cabo un análisis de conglomerados de k-medias con medida de la distancia euclidiana en los factores de AFO de Resistencia No Intrusiva y AFO de Ejercicio Aeróbico para discriminar un grupo de participantes que practicaban la AFO de Resistencia en niveles muy altos en comparación con el resto de la muestra. El factor de AFO de Ejercicio Aeróbico no discriminó entre los grupos, $\mathrm{F}(2,326)=0,11, \mathrm{p}=0,738$, así que tomamos la decisión de llevar a cabo el análisis de conglomerados sólo con el factor de AFO de Resistencia No Intrusiva.

Denominamos los conglomerados como AFO de Resistencia Baja-Normal (Conglomerado 1; $\mathrm{n}=287$ ) y AFO de Resistencia Excesiva (Conglomerado 2; $\mathrm{n}=42$ ). El pequeño tamaño del conglomerado AFO de Resistencia Excesiva es una indicación de la anomalía estadística de este grupo: Como pretendimos, los conglomerados separaban a los individuos que practican niveles excesivos de AFO de Resistencia del resto de la muestra. En la Tabla 2, se muestran las características demográficas por conglomerado. No había diferencias significativas entre los conglomerados según las pruebas chi-cuadrado y t: El valor de $\mathrm{P}$ más bajo fue $p=0,320$. La Tabla B.1 muestra las diferencias entre los conglomerados en la participación en cada AFO.

Tabla B.1 Práctica de Actividad Física de Ocio (AFO) en Horas por Semana por Conglomerado distinguido en la AFO de Resistencia (N = 329)

\begin{tabular}{|c|c|c|c|c|c|c|c|c|}
\hline \multirow[b]{2}{*}{ AFO } & \multicolumn{4}{|c|}{ Conglomerado 1: AFO de Resistencia Baja-Normal $(n=287)$} & \multicolumn{4}{|c|}{ Conglomerado 2: AFO de Resistencia Excesiva $(n=42)$} \\
\hline & $n$ & $\%$ & $M d n$ & $I Q R$ & $n$ & $\%$ & $M d n$ & $I Q R$ \\
\hline Correr & 90 & 31,4 & 1,50 & $2,00-1,00$ & 30 & 71,4 & 4,00 & $6,00-2,00$ \\
\hline Fútbol & 67 & 23,3 & 2,00 & $5,00-1,00$ & 11 & 26,2 & 2,00 & $3,00-1,00$ \\
\hline Culturismo & 64 & 22,3 & 5,00 & $7,50-3,00$ & 8 & 19,0 & 4,92 & $7,13-3,13$ \\
\hline Gimnasia en casa & 33 & 11,5 & 1,00 & $1,50-0,58$ & 25 & 59,5 & 3,00 & $4,75-2,67$ \\
\hline Ciclismo & 43 & 15,0 & 2,00 & $3,00-1,00$ & 11 & 26,2 & 4,50 & $9,67-3,00$ \\
\hline Gimnasia en el gimnasio & 40 & 13,9 & 3,00 & $7,40-1,75$ & 6 & 14,3 & 5,25 & $6,50-2,50$ \\
\hline Pádel & 38 & 13,2 & 1,50 & $2,50-1,00$ & 7 & 16,7 & 1,00 & $1,50-1,00$ \\
\hline Natación & 24 & 8,4 & 2,00 & $4,50-1,13$ & 4 & 9,5 & 3,00 & $4,00-0,64$ \\
\hline Deportes de invierno & 19 & 6,6 & 3,50 & $8,00-2,00$ & 6 & 14,3 & 5,38 & $9,00-2,19$ \\
\hline Baloncesto & 20 & 7,0 & 3,50 & $4,50-1,50$ & 4 & 9,5 & 2,75 & $4,00-0,75$ \\
\hline Tenis & 20 & 7,0 & 2,00 & $3,75-1,00$ & 3 & 7,1 & 1,50 & $--1,00^{\mathrm{a}}$ \\
\hline Atletismo & 14 & 4,9 & 1,63 & $2,63-1,00$ & 1 & 2,4 & 2,50 & $---^{a}$ \\
\hline Patinaje & 11 & 3,8 & 4,00 & $10,50-1,00$ & 4 & 9,5 & 1,00 & $3,25-0,63$ \\
\hline
\end{tabular}

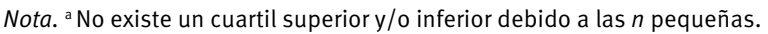




\section{Apéndice $C$ \\ Análisis preliminares para los factores de confusión demográficas}

Se utilizó un análisis correlacional para identificar los potenciales factores de confusión demográficos. Edad correlacionaba significativamente con los PARs relacionados con Internet, $r=-0,15, p=0,007$. Años de educación presentaba asociaciones significativas con las AFOs de fútbol, $r$ $=-0,12, \mathrm{p}=0,032$, $\mathrm{y}$ de gimnasia en el gimnasio, $\mathrm{r}=0,12, \mathrm{p}$ $=0,028$, así como con los PARs relacionados con la alimentación $\mathrm{r}=0,11, \mathrm{p}=0,045$. El nivel educativo completado por la madre correlacionaba con las AFOs de ciclismo, $r$ $=0,12, \mathrm{p}=0,029, \mathrm{y}$ de deportes de invierno, $\mathrm{r}=0,11, \mathrm{p}=$ 0,048 .

Los análisis de varianza mostraron diferencias significativas entre los estudiantes de la facultad de Ciencias del Deporte $(n=125)$ y otras facultades $(n=204)$. Los estudiantes de la facultad de Ciencias del Deporte mostraron medias mayores en la práctica del fútbol, $\mathrm{F}(1,326)=26,13$, $\mathrm{p}<0,001$, el culturismo, $\mathrm{F}(1,326)=29,45$, $\mathrm{p}<0,001$, el pádel, $\mathrm{F}(1,326)=27.23, \mathrm{p}<0,001$, la natación, $\mathrm{F}(1,326)=$ $6.68, \mathrm{p}=0,01$, el baloncesto, $\mathrm{F}(1,326)=7,43, \mathrm{p}=0,007$, el tenis, $\mathrm{F}(1,326)=15,3, \mathrm{p}<0,001$, y el atletismo, $\mathrm{F}(1,326)=$ $3.93, \mathrm{p}=0,048$, y medias menores en los PARs relacionados con la alimentación, $\mathrm{F}(1,326)=5,72, \mathrm{p}=0,017$. También se encontraron diferencias significativas entre los varones $(\mathrm{n}=183)$ y las mujeres $(\mathrm{n}=146)$. Confirmando informes anteriores, los estudiantes varones obtuvieron medias mayores en la práctica de las AFOs del fútbol, $\mathrm{F}(1,326)=30,4$, $\mathrm{p}<0,001$, del culturismo, $\mathrm{F}(1,326)=50,08, \mathrm{p}<0,001$, del ejercicio aeróbico, $\mathrm{F}(1,326)=11,43, \mathrm{p}=0,001$, del pádel, $\mathrm{F}(1,326)=12,49, \mathrm{p}<0,001$, del baloncesto, $\mathrm{F}(1,326)=$ $5,43, \mathrm{p}=0,02$, de los deportes de invierno $\mathrm{F}(1,326)=4,74$, $\mathrm{p}=0,03$, del tenis, $\mathrm{F}(1,326)=7,48, \mathrm{p}=0,007 \mathrm{y}$ del patinaje, $\mathrm{F}(1,326)=5,21, \mathrm{p}=0,023$, así como en los PARs relacionados con videojuegos, $\mathrm{F}(1,326)=30,71, \mathrm{p}<0,001$, el sexo, $\mathrm{F}(1,326)=15,0, \mathrm{p}<0,001$, las apuestas, $\mathrm{F}(1,326)=4,58, \mathrm{p}$ $=0,033$, y medias más bajas en los PARS relacionadas con la alimentación, $\mathrm{F}(1,326)=8,01, \mathrm{p}=0,005$.

En base a estos resultados, en los análisis finales, establecimos medidas de control para las variables edad, género, estudiar o no en la facultad de Ciencias del Deporte, años de educación y nivel de educación completado por la madre, pero no para el nivel de educación completado por el padre. 